\title{
Surproduction ou sous-consommation laitière et santé publique
}

\author{
par \\ W. P. DE STOUTZ, ingénieur \\ et Suzanne BAROI, diététicienne
}

L'économie laitière dans les pays occidentaux et tout particulièrement dans ceux du Marché Commun, semble avoir perdu de vue le rôle qu'elle se doit de jouer dans le domaine alimentaire comme dans celui de la santé publique.

Il n'est question dans les milieux agricoles que de surproduction de lait, de beurre, de lait en poudre, etc., et les responsables gouvernementaux, pour ne pas mécontenter les producteurs, sont contraints de prélever des sommes importantes auprès des contribuables, pour financer les surplus laitiers.

Aussi bien en France que dans l'ensemble du Marché Commun, comme des autres pays occidentaux, la politique semble ignorer l'énorme importance pour la santé publique du facteur nutritionnel que représentent le lait et les produits laitiers.

Ce désintéressement entraîne des dépenses de Sécurité Sociale et de frais sociaux se chiffrant par des centaines de milliards.

Les enquêtes de l'I.N.S.E.R.M. (Institut National de la Santé et de la Recherche Médicale) dans certains départements tests, se traduisent par une moyenne générale, pour la France, de 120 millions d'affections permanentes soit : toutes affections, par habitant : 2,3, et affections graves par habitant : 1,58 (chiffres de 1969 sans doute inférieurs à ceux actuels).

Certes, la thérapeutique a fait des progrès considérables ces dernières décades, notamment en ce qui concerne les maladies infectieuses, mais elle semble impuissante en ce qui concerne les grandes maladies de la civilisation: cancer, affections cardio-vasculaires, rachitisme, etc.

Si l'on est en droit de penser comme le professeur Pech ( Espoir de longue vie ", chez Gallimard) que l'utilisation excessive des pesticides, des défoliants, aussi bien que des antibiotiques, employés 
pour intensifier la production agricole et l'accélération de la croissance des animaux de boucherie et de basse-cour, intervient dans la genèse de ces fléaux sociaux, par le jeu normal de l'alimentation, il est d'autant plus important de mettre en œuvre les avantages pour la santé psychique et physique des individus qu'une consommation rationnelle de lait et de produits laitiers peut apporter.

\section{“ LA LOI DE L'ALIMENT »}

La science atomique nous apprend qu'au regard de la loi de l'unité de la matière, l'ultime division de tout ce qui est, sur et dans la terre, comme dans l'ensemble du cosmos, air, eau, rayonnements solaires, minéraux, végétaux, insectes, cellules, parfums, garçons, filles, aboutit à ce que les américains appellent "Quark " et Louis de Broglie " énergie magnétique ", ou très poétiquement " grains de lumière ».

Ensuite d'actions et d'interactions d'une prodigieuse complexité, ces grains de lumière, ordonnés par un divin chef d'orchestre, que M. Monod appelle "Hasard », deviennent photons, neutrons, atomes, composent les cellules vitales avec leurs différents codes génétiques. Démocrite, prodigieux précurseur, disait voici 2500 ans :

"Les différences entre les atomes appellent des combinaisons et des répulsions. Tout est régi par la nécessité, action naturelle des causes inhérentes ".

La vie est dépendante de la partition des cellules initiales qui font les végétaux, les bactéries, les insectes, les animaux, les garçons, les filles (quarante-sept partitions pour un être humain).

Mais, ces cellules sont soumises tout au long de la vie, à la dégradation irréversible du temps, à une nécessité impérieuse de rééquilibre, au remplacement des "grains de lumière " usés. (Loi de l'entropie).

\section{ENERGIE SOLAIRE EN CONSERVE}

Notre système cellulaire exige donc, pour assurer son autoconservation, autoreproduction et autorégulation, un apport régulier de grains de lumière.

Cette énergie a une source initiale unique, celle des rayonnements solaires, desquels, par le jeu de la photosynthèse, découlent les aliments végétaux, desquels découlent nos aliments animaux, qui sont donc bien des énergies solaires en conserve.

Toutefois, certaines énergies solaires, notamment les plus courtes longueurs d'ondes, ne parviennent à notre torrent sanguin qu'ensuite 
de leur absorption par la surface de notre peau, de notre système pileux.

Il s'agit notamment des précieuses vitamines $\mathrm{D}$, qui assurent entre autres notre équilibre calcium-phosphore, l'harmonieux développement de notre squelette, la prophylaxie du rachitisme.

Dans les régions septentrionnales, le port du vêtement, la vie dans les maisons, les usines, les bureaux, implique donc l'obligation d'absorber par voie buccale les précieux éléments et vitamines complémentaires. Il se trouve que le lait contienne des provitamines D susceptibles d'être synthétisées par l'irradiation.

\section{LA LOI DES EQUILIBRES ALIMENTAIRES}

Mais ces grains de lumière, ces énergies de rééquilibre, doivent être absorbées d'une façon parfaitement équilibrée.

Nous devons à nos maîtres, Mme Lucie Randoin et M. H. Simmonet, cette loi qui se substitue à l'instinct nutritionnel dont bénéficient les animaux à l'état sauvage. C'est à quoi pensait sans doute Bossuet en écrivant :

"La politique de l'homme consiste à tâcher d'égaler les animaux, à qui la Nature a donné la nourriture, le vêtement et le couvert ».

\section{ECONOMIE LAITIERE}

Dès après l'invention de l'élevage au Néolithique, nos ancêtres ont compris l'énorme intérêt nutritionnel du lait. La consommation de lait frais, acidifié, divers, dans les tribus d'éleveurs, était de plusieurs litres par jour, apportant une part importante des calories indispensables, toutes les protéines nécessaires aux synthèses du métabolisme, de même que les matières grasses, hydrates de carbone, les vitamines essentielles, y compris les vitamines $\mathrm{D}$, captées par la peau et les poils des animaux, les minéraux et les oligoéléments, le calcium et le phosphore, en quantité suffisante et avec une valeur biologique optimale.

L'histoire nous apprend que, sans exception, les nomades éleveurs, grâce à leur vitalité, leur énergie, leur intelligence, ont dominé et conquis les tribus sédentaires. Ainsi en fut-il des Chinois par les Mongols, des Ligures de la Gaule par les Celtes, puis de ces mêmes Celto-romains sédentarisés par les Germains de Clovis. Plus près de nous, les défaites des Bourguignons sédentaires par les éleveurs des cantons suisses, illustrent cette extraordinaire vitalité. 
Il se trouve que la « loi des équilibres alimentaires » a situé en moyenne générale la consommation de lait et de produits laitiers par jour à $0,600 \mathrm{l}$ de lait frais pasteurisé, $120 \mathrm{~g}$ de fromages divers, et $36 \mathrm{~g}$ de beurre, ce qui représente en équivalent lait environ 2,8 1 par jour.

Il se trouve que les statistiques du Centre National Interprofessionnel de l'Economie Laitière C.N.I.E.L., nous donne « per capita » une consommation moyenne annuelle de 55,6 1 - consommation à la ferme non comprise - 0,36 $\mathrm{kg}$ de crème, $9,3 \mathrm{~kg}$ de beurre, $15,4 \mathrm{~kg}$ de fromage, qui nous donne en équivalent lait 1,2201 par jour.

La consommation de lait est donc insuffisante de $(2,8001$ $1,220)=1,5801$ par habitant et par jour en moyenne générale. Il n'y a donc pas, il ne peut y avoir de surplus laitiers, mais bien une importante sous-consommation, au regard de la loi des équilibres alimentaires.

Les conséquences d'une politique de redressement de cette situation sont considérables pour l'ensemble de l'économie de la Nation, en ce qu'elles impliquent un doublement du cheptel bovin actuel, par voie de conséquence la création de quelque 700000 fermes familiales de 14 têtes, la résorption définitive du chômage.

La distribution généralisée et gratuite du lait dans les écoles, qui doit être à la base de cette action, comme les frais d'investissement nécessaires, sont rapidement récupérables sur les économies de frais de Sécurité Sociale et des frais sociaux, si l'on considère qu'une économie moyenne sur ces divers frais, de $10 \mathrm{~F}$ par habitant et par jour, représente 190 milliards de $\mathrm{F}$ par an.

\section{CONCLUSION}

L'application des règles de la nutrition équilibrée nécessite le doublement de la production laitière. Les conséquences pour l'économie agricole, comme pour l'état de santé de la population, sont incalculables ; une telle politique implique ipso facto la suppression du chômage, un climat social équilibré. ce but.

Bien évidemment, il faut un effort d'organisation pour atteindre

Bien entendu, les détracteurs vont être innombrables. Toutefois, aucun ne peut apporter une contradiction scientifique sérieuse. 